\title{
Stability of spin droplets in realistic quantum Hall devices
}

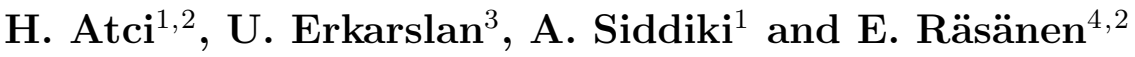 \\ ${ }^{1}$ Physics Department, Faculty of Science, Istanbul University, 34134 Vezneciler \\ Istanbul, Turkey \\ ${ }^{2}$ Nanoscience Center, Department of Physics, University of Jyväskylä, FI-40014 \\ Jyväskylä, Finland \\ ${ }^{3}$ Department of Physics, Faculty of Science, Mugla University, 48170 Kötekli-Mug̃la, \\ Turkey \\ ${ }^{4}$ Department of Physics, Tampere University of Technology, FI-33101 Tampere, \\ Finland \\ E-mail: huseyinatci@gmail.com
}

\begin{abstract}
We study the formation and characteristics of "spin droplets",i.e., compact spin-polarized configurations in the highest occupied Landau level, in an etched quantum Hall device at filling factors $2 \leq \nu \leq 3$. The confining potential for electrons is obtained with self-consistent electrostatic calculations on a GaAs/AlGaAs heterostructure with experimental system parameters. Real-space spin-density-functional calculations for electrons confined in the obtained potential show the appearance of stable spin droplets at $\nu \sim 5 / 2$. The qualitative features of the spin droplet are similar to those in idealized (parabolic) quantum-dot systems. The universal stability of the state against geometric deformations underline the applicability of spin droplets in, e.g., spin-transport through quantum point contacts.
\end{abstract}

PACS numbers: 73.43.Cd, 73.21.La 


\section{Introduction}

Recent research on semiconductor quantum dots (QDs) has strongly focused on spin effects due to experimental breakthroughs in the initialization, control, and readout of spin states that have decoherence times up to milliseconds [1]. Consequently, QDs are among the leading candidates for solid-state qubit design. On the other hand, studies on few-electron QDs in strong magnetic fields have shown interesting similarities to partially or fully spin-polarized quantum Hall $(\mathrm{qH})$ states in the two-dimensional (2D) electron gas [2] (2DEG). For example, the filling factor $\nu=1$ in the 2DEG corresponds to a "maximum-density droplet" 3] in a few-electron QD. As another example, the Laughlin wave function [4] - describing the filling factor $\nu=1 / 3$ state in the 2DEG was found to have $98 \%$ overlap with the corresponding few-electron QD (three vortices per electron) [5].

The analogy between the 2DEG and QDs applies further to the $\nu=5 / 2$ state, although a direct comparison is more complex. A. Harju etal. [6] showed that halfinteger filling-factor states in QDs correspond to a situation where the highest occupied Landau level (LL) is fully spin-polarized, whereas the lower LLs are spin-compensated. Later on, the spin polarization of the highest LL is shown with three independent spinblockade experiments [7, 8]. Interestingly, these "spin droplets" were found to form only when the number of confined electrons exceeded $\sim 30$ (Ref. [7]). In this respect, spin droplets are collective states induced by a high density of states close to the Fermi level that might lead to collective spin polarization. On the other hand, the correspondence between the highest-LL spin droplets and the candidates of the $\nu=5 / 2$ state in the 2DEG, such as the Pfaffian wave function, was found to be ambiguous [9].

Although numerical studies have shown the appearance of spin droplets also in quantum rings [10], there is no systematic investigation on the stability of those states. This issue is of fundamental importance when the spin droplets will be applied in more general $\mathrm{qH}$ devices such as quantum point contacts [11, 12]. In this work we will focus on this aspect of stability and perform a thorough theoretical investigation on the formation of spin droplets in a realistic $\mathrm{qH}$ device starting from actual device geometry and parameters. Our calculations show that the spin-droplet formation in a realistic device is very similar to that in "idealized", i.e., parabolically (harmonically) confined QDs. Thus, our finding confirms the high stability of spin droplets against geometric deformations. This might have significant consequences for the applicability of those states in, e.g., spintronics.

The paper is organized as follows. In Sec. 2 we first perform self-consistent electrostatic calculations to extract the confining potential for electrons in a realistic $\mathrm{qH}$ device. Then we present the many-electron Hamiltonian and our spin-densityfunctional-theory [13] (SDFT) approach to calculations in the $\mathrm{qH}$ regime. Our results for total energies, total and spin densities, and chemical potentials are presented in Sec. 3. The paper is summarized in Sec. 4. 


\section{Methods}

\subsection{Quantum-dot structure and the confining potential}

We consider a two layer $\delta$-Si doped GaAs/AlGaAs heterostructure provided by Goldman [14] and visualized in Figure 1. Here, the crystal is grown on a GaAs substrate and the 2DEG is formed at the interface of the GaAs/AlGaAs heterostructure located $284 \mathrm{~nm}$ below the surface. The donor layers located $122 \mathrm{~nm}$ and $248 \mathrm{~nm}$ above the 2DEG have surface densities $2.5 \times 10^{15} \mathrm{~m}^{-2}$ and $1.7 \times 10^{16} \mathrm{~m}^{-2}$, respectively. The geometry of the QD is shown on top of the GaAs/AlGaAs heterostructure in Figure 1. The actual sample is obtained with etching $80 \mathrm{~nm}$ from the surface (in the $z$ direction). The 2D dimensions on the $x y$ plane are $L_{x}=L_{y}=2550 \mathrm{~nm}$.

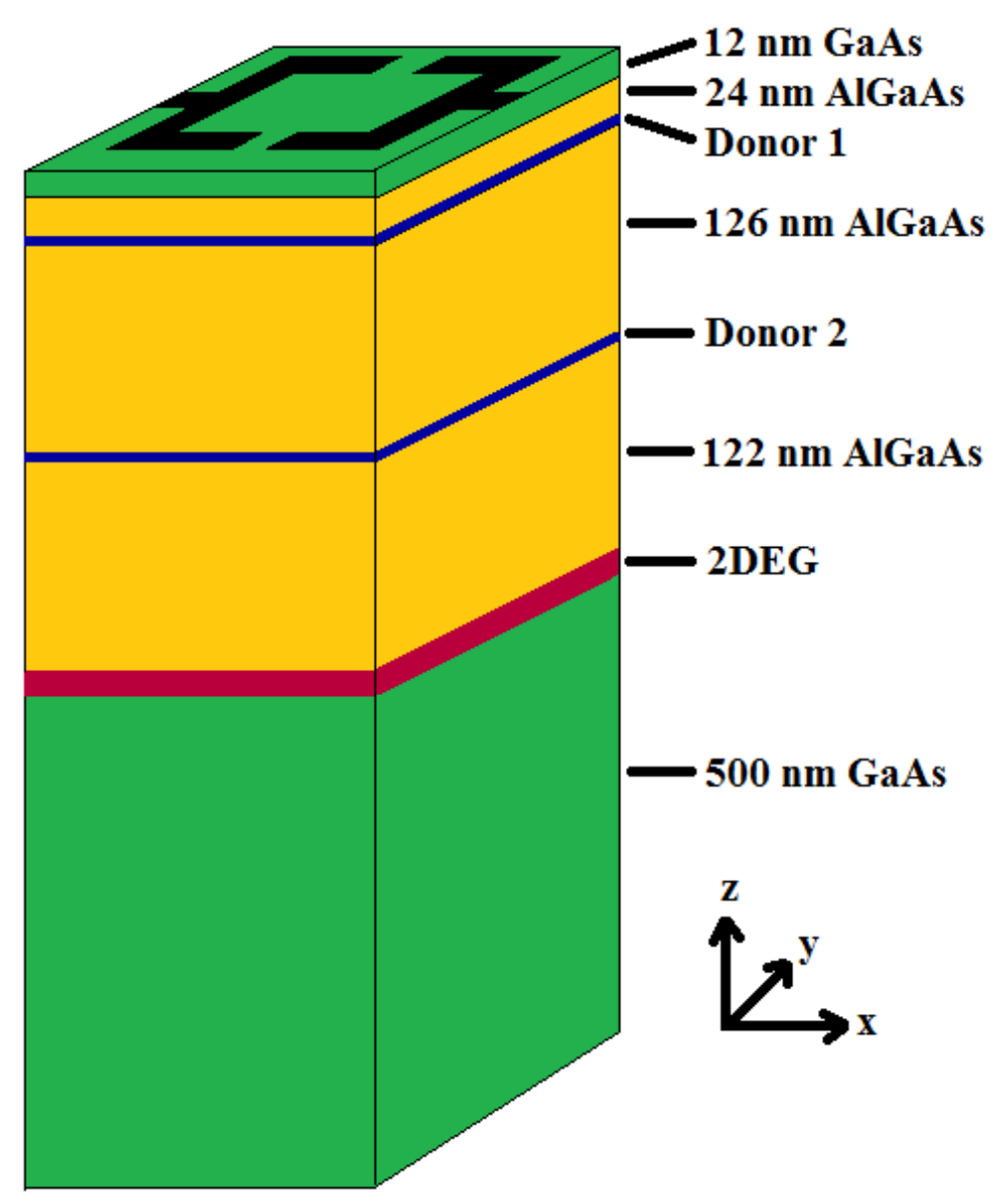

Figure 1. Structure of the $\mathrm{qH}$ device applied in the calculation of the confinement potential. The material parameters are from Goldman [14.

The QD sample is mapped on a matrix with $128 \times 128 \times 32$ mesh points. The confinement profile for the electrons trapped in the 2DEG, i.e., for our QD, is calculated by solving the Poisson equation in 3D self-consistently within the Thomas-Fermi approximation [15, 16]. In the numerical calculation we apply a fourth-order nearest neighbor approximation and a 3D Fourier transformation. Here we apply open boundary 
conditions, since the heterostructure is embedded in a dielectric surrounding with a very small dielectric constant compared to the heterostructure.

The obtained confining potential is shown in Figure ??. It is noteworthy that the potential is not rotationally symmetric in contrast with the commonly used parabolic approximation for both lateral and vertical semiconductor QDs [17]. Moreover, the shallow etching leads to a relatively smooth potential, although the slope of the confinement is still considerably steeper than in a parabolic QD.

In the following we may consider the obtained confining potential in Figure ?? as a trap for transported electrons forming a QD inside the device. Alternatively, we can think that - by adjusting the gate and bias voltages - the 2DEG inside the QD is emptied from conduction-band electrons one-by-one. It is important to note that in the following calculations we focus on such a QD-like system with a few dozen electrons instead of the bulk 2DEG in the $\mathrm{qH}$ regime. Our particular task is to investigate the effect of a relatively sharp and rotationally non-symmetric potential on the formation of spin-droplets in QDs at $\nu \sim 5 / 2$.

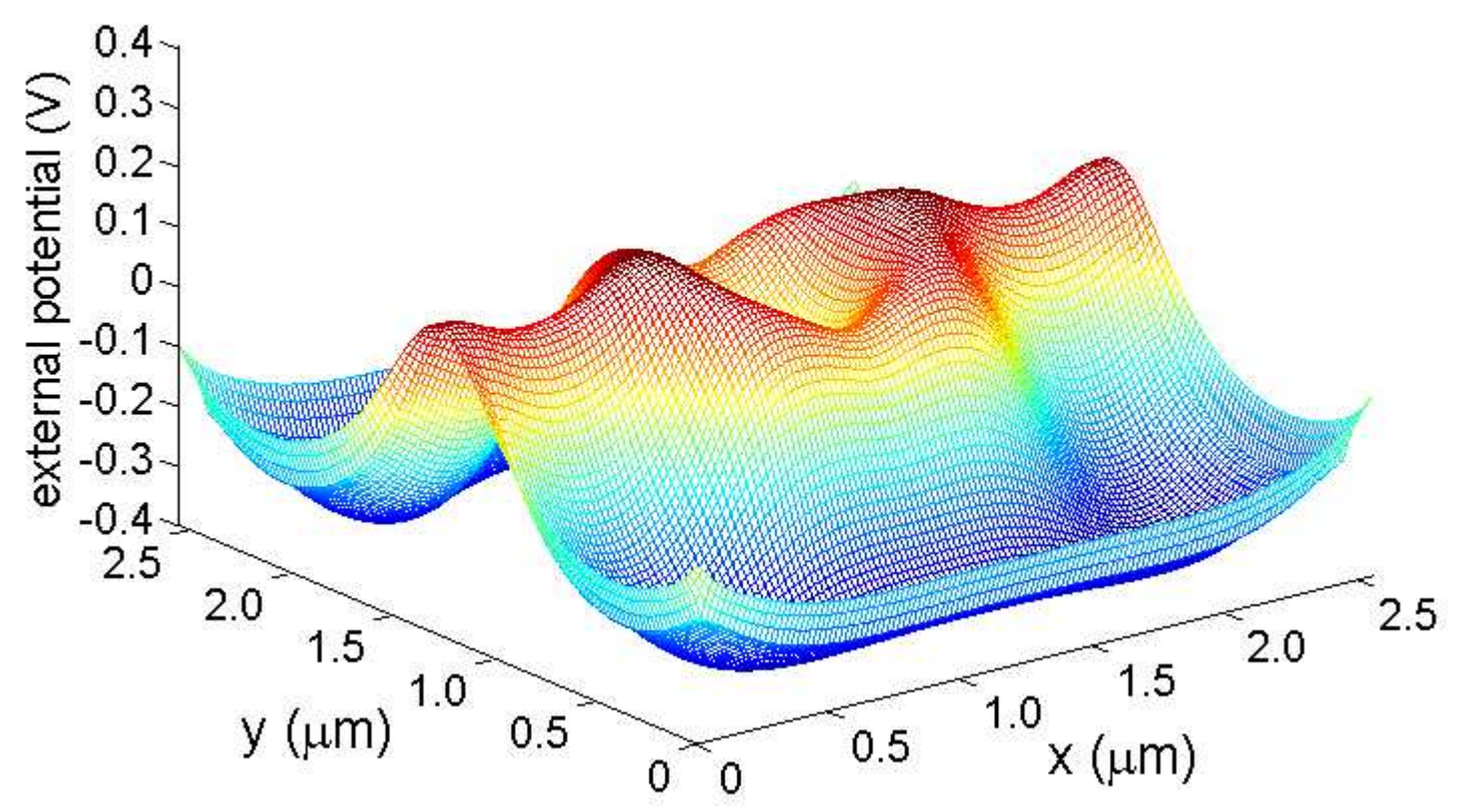

Figure 2. Confining potential for electrons in the QD obtained with electrostatic calculations for the GaAs/AlGaAs heterostructure shown in Figure 1. 


\subsection{Hamiltonian and many-electron calculations}

Electrons in the QD are described by the Hamiltonian

$$
H=\sum_{i=1}^{N}\left[\frac{\left(\mathbf{p}_{i}+e \mathbf{A}\right)^{2}}{2 m^{*}}+V_{c}\left(\mathbf{r}_{i}\right)+g^{*} \mu_{B} S_{z, i}\right]+\frac{e^{2}}{4 \pi \epsilon} \sum_{i<j} \frac{1}{\mathbf{r}_{i j}},
$$

where $N$ is the number of electrons, $V_{c}$ is the confining potential obtained in the previous section (Figure ??), and $\mathbf{A}$ is the vector potential of the homogeneous magnetic field oriented perpendicular to the QD plane. We consider the conventional effective-mass approximation [17] with GaAs material parameters $m^{*}=0.067 m_{e}, \epsilon=12.7 \epsilon_{0}$, and $g^{*}=-0.44$ for the gyromagnetic ratio.

As we consider $N \approx 48$ confined electrons, the many-electron Hamiltonian is not solvable numerically exactly. The non-circular geometry sets additional limitations. Hence, we use SDFT [13] that has been shown to produce reliable results when compared with quantum Monte Carlo (QMC) calculations [7, 9]. An explicit comparison between SDFT and QMC results for spin-state energies can be found in Fig. 8 of Ref. [9]. For the exchange and correlation we use the 2D local-spin-density approximation (LSDA) with the parametrization of Attaccalite et al. [18] for the correlation. In view of the previous works, [7, 8, 9] we rely on the computationally efficient LSDA despite recent progress in the development of alternative and more accurate density functionals for $2 \mathrm{D}$ systems [19, 20, 21].

Our real-space SDFT approach with the octopus code [22] allows the use of the confining potential in Figure ?? as a direct input. We calculate the total energies and spin densities for different spin configurations, respectively, and determine the ground state as the solution with the lowest energy. It should be noted that in lack of rotational symmetry the angular momentum is not a good quantum number. However, the (approximate) angular momenta of the effective single-electron (Kohn-Sham) states enable us to determine the occupations of different LLs (see below).

\section{Results}

\subsection{Total energies}

In the results presented below the magnetic-field range has been selected such that the regime at filling factors $2 \leq \nu \leq 3$ is covered. The filling factor in a QD can be approximated by $\nu \approx 2 N / N_{0 \mathrm{LL}}$, where $N_{\text {OLL }}$ is the number of electrons in the lowest LL [7]. We point out that this approximation is valid only at $\nu \geq 2$, whereas otherwise a good estimate is given by $\nu=N / N_{\Phi_{0}}$, where $N_{\Phi_{0}}$ is the number of flux quanta $\Phi_{0}=e / h$. Detailed comparison between different definitions for the filling factor in confined systems is given in Ref. [10].

The total energy can be written as a sum $E_{\text {tot }}=E_{\text {kin }}+E_{\text {ext }}+E_{H}+E_{x c}$, where $E_{\text {kin }}$ is the (Kohn-Sham) kinetic energy, $E_{\text {ext }}=\int d \mathbf{r} \rho(\mathbf{r}) V_{c}(\mathbf{r})$ is the external confinement energy, $E_{H}$ is the classical electrostatic (Hartree) interaction energy, and 


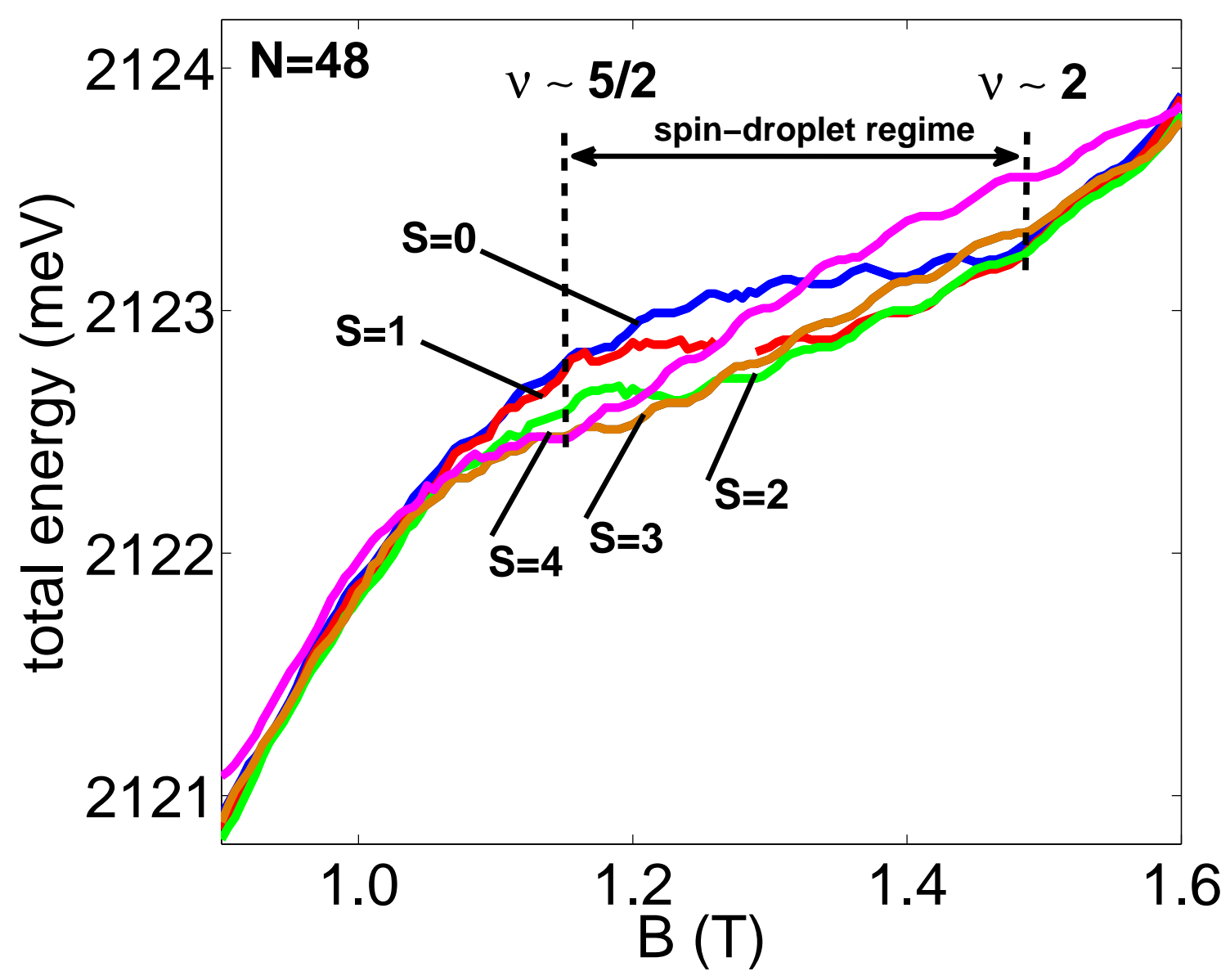

Figure 3. Total energies of different spin states as a function of the magnetic field in a 48-electron QD calculated with SDFT in the spin-droplet regime $(2 \leq \nu \leq 3)$.

$E_{x c}$ is the exchange-correlation energy accounting for quantum-mechanical interaction energy components beyond $E_{H}$. In Figure 3 we show the total energies of different spin states $S$ as a function of the magnetic field for a 48-electron QD. The points of $\nu \sim 5 / 2$ and $\nu \sim 2$ according to the above definition are marked by dashed lines. At $B \leq 1.1$ T the states with different $S$ are almost degenerate until a distinctive energy gap opens up between the higher and lower $S$. The maximum ground-state spin is $S=4$, and as $B$ is increased the polarization of the QD gradually decreases: at $B \geq 1.5 \mathrm{~T}$ the ground state has $S=1$, and the unpolarized $S=0$ state is again very close in energy.

Overall, the behavior of $E_{\text {tot }}$ in Figure 3 is very similar to that of parabolic QDs [7]. In the following we analyze the corresponding spin densities and chemical potentials in detail in order to characterize the expected spin-droplet formation.

\subsection{Spin densities at $\nu \sim 2$ and $\nu \sim 5 / 2$}

In Figure 4 we show the total and spin densities in a 48-electron QD at $B=1.492 \mathrm{~T}$ corresponding to $\nu \sim 2$. The ground-state total spin is $S=1$ as a slight deviation from 

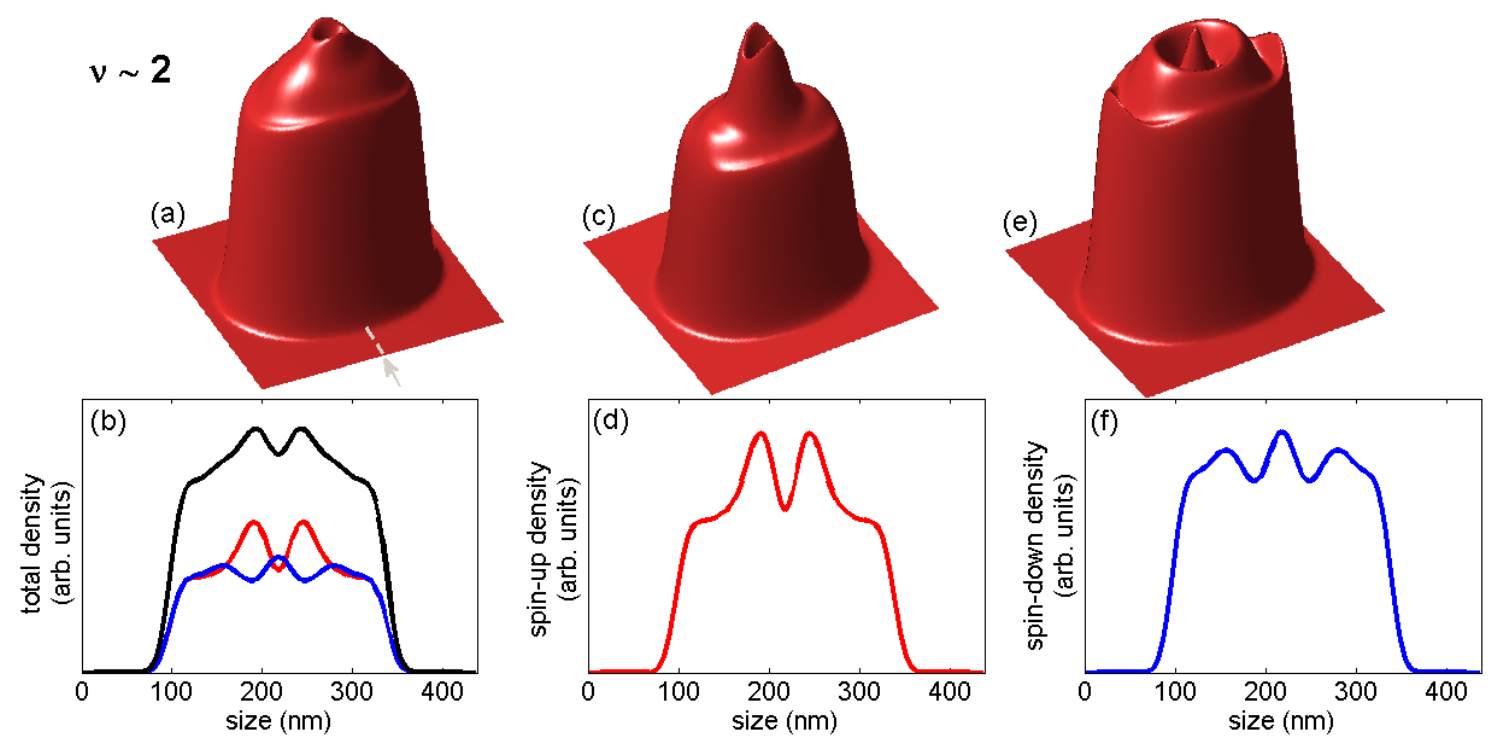

Figure 4. Total and spin densities in a 48-electron QD at $B=1.492 \mathrm{~T}$ corresponding to $\nu \sim 2$. The cross sections in the lower panel are taken along a vertical cut across the $2 \mathrm{D}$ densities in the upper panel. The total spin is $S=1$. (a-b) Total density, (c-d) spin-up density, (e-f) spin-down density.

an ideal $\nu=2$ state, which is fully spin-compensated, i.e., the states in the lowest LL are doubly filled with spin-up and spin-down electrons [7], In our case, it seems clear in Figure 4(b) that the polarized electrons are located close to the core of the QD in the second-lowest Landau level (1LL). Nevertheless, the total density is relatively flat in accordance with the $\nu=2$ state in a parabolic QD. We note that the rotational symmetry is broken due to the non-circular confining potential. The cross sections in the lower panel of Figure 4 are taken along a vertical cut across the 2D densities in the upper panel.

Figure 5 shows the total and spin densities similarly to the previous case but now at $B=1.15 \mathrm{~T}$ corresponding to $\nu \sim 5 / 2$. The total spin is $S=4$. It is interesting to note that the spin polarization is strongly concentrated close to the core of the QD. This is confirmed by sorting the Kohn-Sham states according to their spin and angular momenta. Hence, it is clear that the (eight) polarized electrons occupy the 1LL. We may thus call this state as a spin droplet. Its characteristics are strikingly similar to those in a parabolic QD. Despite the non-circular potential, the density of the spin droplet in the core region [Figures 5(c) and (d)] is very smooth. Most likely, this is a consequence of the screening of the irregularities in the potential by the 0LL electrons, so that the 1LL with the spin droplet has a smooth surrounding potential. It might be expected that the spin droplet could survive in even more irregular geometries, i.e., in the vicinity of a quantum point contact. This aspect of universality deserves more investigations.

An important aspect in the formation of a spin droplet is the sufficient $N$ for the phenomenon to occur. In parabolic QDs spin droplets at $2 \leq \nu \leq 3$ were found 


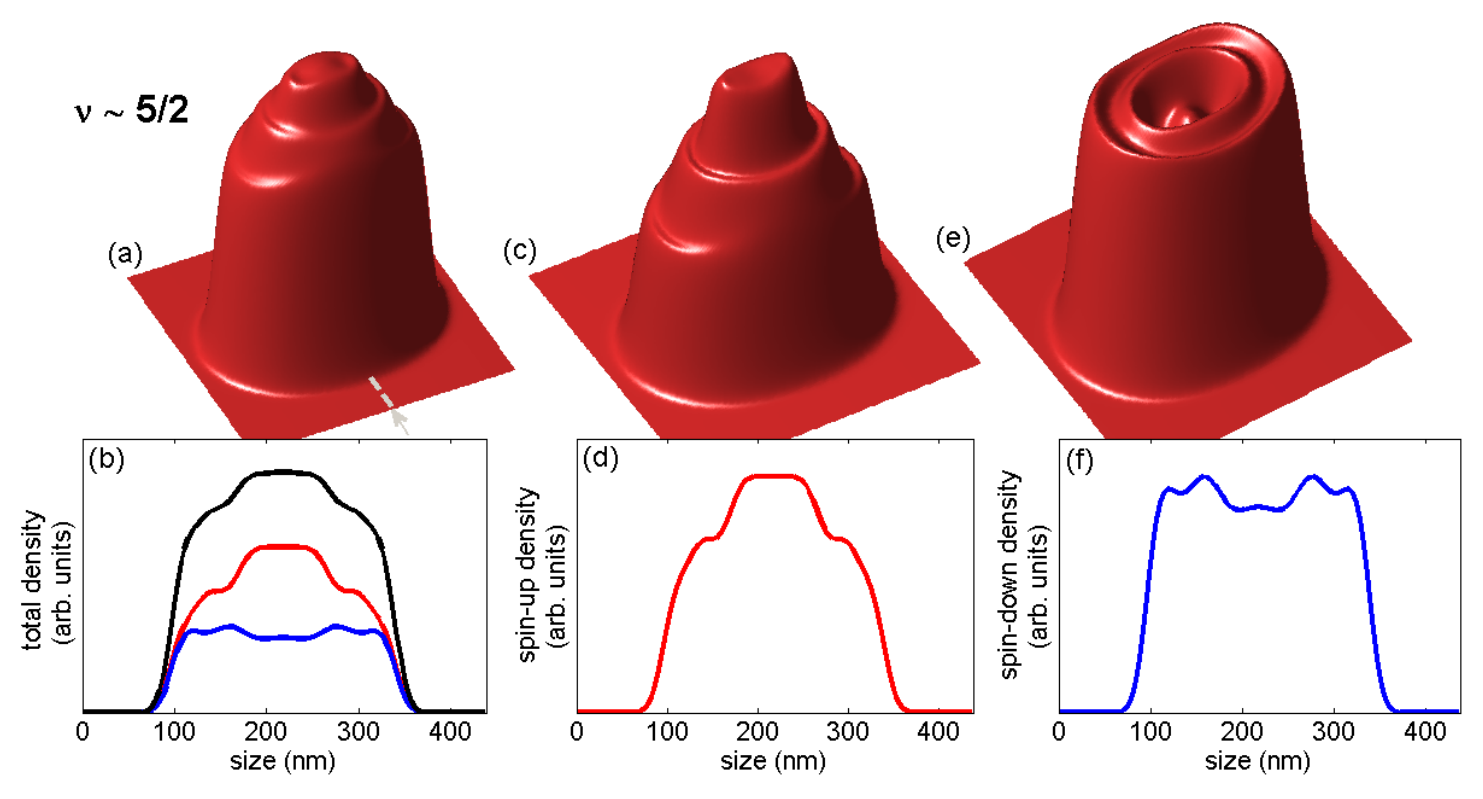

Figure 5. Total and spin densities in a 48-electron QD at $B=1.15 \mathrm{~T}$ corresponding to $\nu \sim 5 / 2$. The cross sections in the lower panel are taken along a vertical cut across the $2 \mathrm{D}$ densities in the upper panel. The total spin is $S=4$. (a-b) Total density, (c-d) spin-up density, (e-f) spin-down density.

when the number of electrons was $N \geq 30$. According to our calculations there is no considerable change in the critical $N$ in a non-circular geometry, so that the droplet emerges at $N \approx 30$ as well. As discussed in Sec. ?? the spin polarization of the highest occupied LL is a collective effect resulting from a high density of states close to the Fermi energy. This is the case if (i) $N$ is sufficiently large and (ii) a proper fraction of the electrons occupy the highest LL (for $\nu \sim 5 / 2$ the highest LL is the second one). The effect resembles Hund's rule: at the expense of kinetic energy, spin polarization saves exchange energy close to a point of degenerate states. Here, with dozens of electrons involved in the process, the phenomenon is similar to the Stoner effect [23], which predices the emergence of ferromagnetic alignment in a correlated electron system if the degeneracy is high close to the Fermi level.

\subsection{Chemical potentials in the spin-droplet regime}

Finally we consider the chemical potentials to assess the signals that the formation of spin droplets in leave in spin-blockade oscillations. The chemical potential is defined as $\mu(N)=E_{\text {tot }}(N)-E_{\text {tot }}(N-1)$. Here we consider $\mu(48)$ by computing the energies of different spin states for $N=47$ and $N=48$. For both systems, respectively, the lowest energy state (ground-state) was chosen to calculate the chemical potential.

Figure 6] shows the chemical potential as a function of the magnetic field in the vicinity of the spin-droplet range. We find a clear "plateau" region at $2 \leq \nu \leq 5 / 2$ superimposed by distinctive sawtooth-like oscillations. The structure is similar to the 


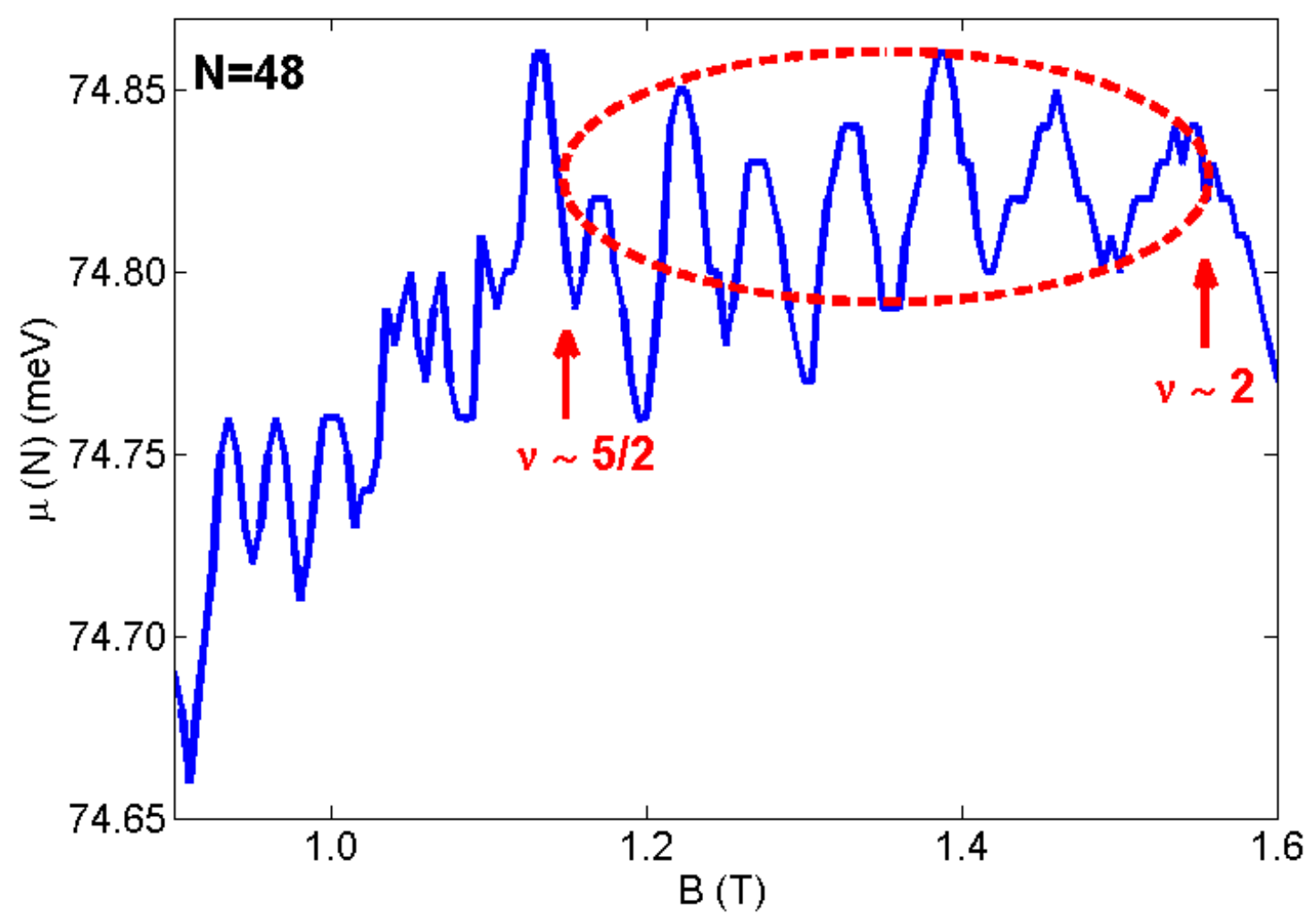

Figure 6. Chemical potential for a 48-electron QD as a function of the external magnetic field. The spin-droplet regime is marked with a dashed ellipse.

Coulomb-blockade peak oscillations measured in both lateral and vertical QDs [7, 8, 9, Moreover, the qualitative features are similar to the previous SDFT calculations for parabolic QDs [7], although in this case we find more irregularities. In fact, the irregularities in $\mu$ resemble the experimental data; it might be expected that in actual QDs the impurities etc. induce similar effects found here for a non-circular qH device.

\section{Summary}

In summary, we have studied the spin-droplet formation in realistic quantum Hall devices in magnetic fields corresponding to filling factors $2 \leq \nu \leq 3$. We carried out self-consistent electrostatic calculations for a GaAs/AlGaAs heterostructure with experimental parameters. In this way we obtained a confining potential for electrons that can be trapped inside the quantum dot. Our spin-density-functional-theory studies for $\sim 48$ interacting electrons in the determined non-circular potential show that (i) spin polarization occurs at $\nu \sim 5 / 2$, (ii) polarized electrons are located at the core of the dot in the second Landau level, (iii) the spin droplet is very smooth and thus similar to that in an ideal parabolic quantum dot, (iv) the critical $N$ for the formation of the spin droplet is not affected by having a realistic (non-symmetric) potential, and (v) the spin-droplet formation leaves a signal to the chemical potential that resembles the 
available experimental spin-blockade data.

We hope that the present study encourages further experimental studies on the subject. In particular, it would be important to detect the spin-droplet directly in an experiment by appropriate spin-imaging methods. In addition, although the present study confirms the stability of the spin droplet in a non-circular geometry, the state is still to be found in a large system with $N \sim 1000$ confined electrons, where it can be assumed to be extremely stable. The ongoing experiments on quantum point contacts are likely to bring answers to these assumptions in near future, and they may open up the path for further applications.

\section{Acknowledgments}

We are grateful to V. J. Goldman for providing us with the layer structure. This work was supported by the Academy of Finland (HA and ER), Wihuri Foundation (ER), ERASMUS Internship Programme (HA), the scientific and technological research council of Turkey (TÜBITAK) under grant no:TBAG-109T083 (HA and AS), IUBAP:6970 (AS) and Institute of Theoretical and Applied Physics (ITAP) in Turunç, Turkey. CSC Scientific Computing Ltd. is acknowledged for computational resources.

\section{References}

[1] For a review, see Hanson R, Kouwenhoven L P, Petta J R, Tarucha S and Vandersypen L M K 2010 Rev. Mod. Phys. 791217

[2] Giuliani G F and Vignale G 2005 Quantum Theory of the Electron Liquid (New York: Cambridge University Press)

[3] MacDonald A H, Eric Yang S-R and Johnson M D 1993 Aust. J. Phys. 46345

[4] Laughlin R B 1983 Phys. Rev. B 273383

[5] Saarikoski H, Harju A, Puska M J and Nieminen R M 2004 Phys. Rev. Lett. 93116802

[6] Harju A, Saarikoski H and Räsänen E 2006 Phys. Rev. Lett. 96126805

[7] Räsänen E, Saarikoski H, Harju A, Ciorga M, and Sachrajda A 2008 Phys. Rev. B 77 041302(R)

[8] Rogge M, Räsänen E and Haug R J 2010 Phys. Rev. Lett. 105046802

[9] Saarikoski H, Tölö E, Harju A and Räsänen E 2008 Phys. Rev. B 78195321

[10] Räsänen E and Aichinger M 2009 J. Phys.: Condens. Matt. 21025301

[11] Dolev M, Heiblum M, Umansky V, Stern A and Mahalu D 2008 Nature 452829

[12] Dolev M, Gross Y, Heiblum M, Umansky V, Stern A and Mahalu D 2010 AIP Conf. Proc. 1199 239

[13] For a review on density-functional theory, see, e.g., Dreizler R M and Gross E K U 1990 Density Functional Theory (Berlin: Springer)

[14] Camino F E, Zhou W and Goldman V J 2005 Phys. Rev B 72155313

[15] Arslan S, Cicek E, Eksi D, Aktas S, Weichselbaum A and Siddiki A 2008 Phys. Rev. B 78125423

[16] Siddiki A and Gerhadts R R 2003 Phys. Rev. B 68125315

[17] Reimann S M and Manninen M 2002 Rev. Mod. Phys. 741283

[18] Attaccalite C, Moroni S, Gori-Giorgi P and Bachelet G B 2002 Phys. Rev. Lett. 88256601

[19] For recent works, see, e.g., Räsänen E, Pittalis S and Proetto C R 2010 Phys. Rev. B 81 195103; Pittalis S and Räsänen E 2010 Phys. Rev. B 82 165123; Pittalis S and Räsänen E 2010 Phys. Rev. B 82 195124; Pittalis S, Räsänen E, Vilhena J G and Marques M A L 2009 Phys. Rev. A 79012503 
[20] Constantin L A 2008 Phys. Rev. B 78155106

[21] Constantin L A, Perdew J P and Pitarke J M 2008 Phys. Rev. Lett. 101016406

[22] Castro A, Appel H, Oliveira M, Rozzi C A, Andrade X, Lorenzen F, Marques M A L, Gross E K U and Rubio A 2006 Phys. Stat. Sol. (b) 2432465

[23] Stoner E C 1938 Proc. Roy. Soc. A 165372 